\title{
Analysis of Parameters Influence on the Characteristics of Thomson Coil Type Actuator of Arc Eliminator Using Adaptive Segmentation Equivalent Circuit Method
}

\author{
Wei Li*, Young Woo Jeong**, Hee Sung Yoon* and Chang Seop Koh ${ }^{\dagger}$
}

\begin{abstract}
A Thomson coil type actuator is applied as the driving unit in an arc eliminator system. To eliminate the arc efficiently, the speed of the actuator is required as fast as possible with certain limit of the exciting current. Therefore, the dynamic characteristics of the Thomson coil type actuator should be analyzed in an effective way. In this paper, a novel solving technique has been developed based on the equivalent circuit model which is set up by dividing the conducting plate into multi segments. To guarantee the calculation accuracy and improve the calculation efficiency, an adaptive refinement algorithm is suggested based on the field continues condition. The proposed method has been verified by the FEM calculation and experiment. The influence of circuit and plate parameters to the performance of the actuator is also investigated, from which a reasonable set of parameters can be found.
\end{abstract}

Keywords: Thomson coil type actuator, Arc Eliminator, Adaptive segmentation, Equivalent circuit model

\section{Introduction}

An arc due to a severe fault in a switchgear can result in a series of damage if the arcing time is long, such as high temperature, high pressure, and poisonous gases. Therefore, to feed the requirement of high service reliability and safety for personnel, a device named arc eliminator is developed for extinguishing the arc by bypassing the power current from the distribution line to a ground with a very high speed action in terms of just a few milliseconds. A short acting time means that no significant amount of energy has time to build up in the arc, therefore, no damage will be caused. For this reason, a fast response driving actuator is required to be taken as the driving unit of the arc eliminator.

Until now, several kinds of mechanism have been employed as the driving actuator, such as spring mechanism, electromagnetic actuator, linear motor driven mechanism and Thomson coil type actuator. Compare to other mechanisms, the Thomson coil type actuator utilizing the repulsion force between the exciting coil and the conducting plate responses much faster. Therefore, it is quite suitable to apply the Thomson coil type actuator in the arc eliminator system.

For the dynamic performance analysis of the Thomson coil type actuator, finite element method (FEM) is the most popular method. Although FEM is proven precise to solve this kind of problems, however, it is quite time consuming.

$\dagger$ Corresponding Author: Dept. of Electrical Engineering, Chungbuk National Univerity, Korea. (kohcs@chungbuk.ac.kr)

* Dept. of Electrical Engineering, Chungbuk National Univerity, Korea. (liwei@chungbuk.ac.kr, hsyoon@chungbuk.ac.kr)

* Switchgear Research Team, Electrotechnology, R\&D Center, LSIS, Co., Ltd., Cheongju, Chungbuk 361-720, Korea. (ywjeong@1sis.biz) Received: June 2, 2009; Accepted: March 9, 2010
As there is no magnetic material in the Thomson coil type actuator, it is possible to analyze the property by using analytic equations, which is much more efficient than using FEM. Some researches about the numerical calculation of this kind electromechanical coupled problem using analytic equations have been done [1]-[3]. The key points of solving these kind problems accurately are the construction of equivalent model and the evaluation of parameters, here mainly means the calculation of inductance.

In this paper, a more accurate and efficient algorithm for analyzing the dynamic characteristics of Thomson coil type actuator is proposed. In the algorithm, the whole system is transferred into an equivalent circuit model by considering the influence of the eddy current. The repulsion plate is divided into many segments using adaptive segmentation method according to the distribution of eddy current, and each segment corresponds to one circuit with parameters of resistance, self inductance and mutual inductance with other circuits. The inductance is calculated by using an algorithm based on Bartky's transformation [4]. The state equations that abstract from the equivalent circuit model are solved by applying Runge-Kutta-Fehlberg method. This novel solving technique is tested by analyzing the dynamic characteristics of a high speed Thomson coil type actuator. By comparing with the FEM calculation results and experiment results, the proposed method is proven more accurate and efficient, at the same time, based on which, the analysis of the dynamic performance of the arc eliminator is done. Furthermore, the influence on the performance from different parameters including both the circuit parameters and the plate parameters are analyzed, through which the optimal parameters are found. 


\section{Structure of the Arc Eliminator}

Fig. 1 outlines the simplified structure of an arc eliminator employing a Thomson coil type actuator. The driving mechanism is mainly composed of exciting coils corresponding to opening and closing coils, and a moving plate with a contact that usually defined by high conductivity and light weight. The moving range of the plate is the area between the opening and closing coils. The other assistant elements such as the spring for supporting the plate will not be introduced here. The exciting current of the coils are supplied by capacitor bank, and this corresponds to a $R L C$ series circuit as shown in Fig. 2, where, $R c$ represents the inner resistance of the capacitor, and the resistance of the connecting wire is ignored. Switch $\mathrm{S} 1$ will be closed to charge the capacitors to a rated voltage.

In normal case, the normal current will flow along the distribution line, as labeled in Fig. 1, and the plate stays at the open position. At this moment, the fixed contact and the moving contact are separated; therefore, there is no fault current flowing through the fixed and moving contacts. If an open arc due to a severe fault in the switchgear is detected by the arc monitoring system, then a trip signal is sent to close switch $S_{2}$, due to which a pulse current will be generated in the $R L C$ series circuit that will result in eddy current in the conducting plate. The electromagnetic repulsive force generated between the exciting coil and plate will drive the plate together with the moving contact away to the close position, at which the conducing path of fault current is constructed by the connection of fixed contact and moving contact. Therefore, the fault current will be bypassed to the ground through the conducting path. As the arcing time is very short, it will not cause any high pressure or any thermal damage at all. The arc eliminator will be reset by the opening coil after the fault is cleared.

It is important to generate a large repulsive force in short time, which will lead to a high speed of the plate and shorten the time of action. Therefore, step by step analysis

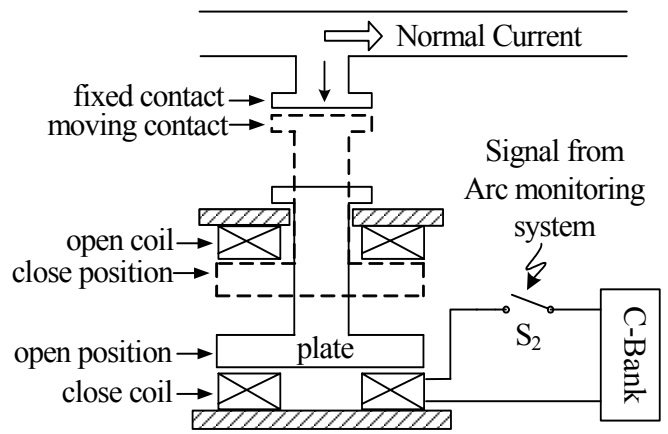

Fig. 1. The simplified mechanism of the arc eliminator.

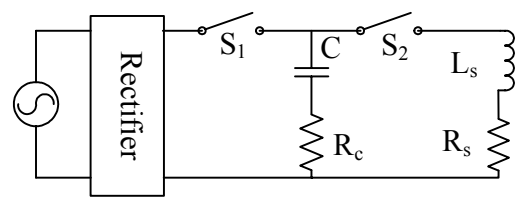

Fig. 2. The circuit of the exciting coil. of the transient electromagnetic field taking into consideration of the friction and load force is required to evaluate the drive efficiency of the actuator, as well as the influence of parameters such as circuit parameters and plate dimensions.

\section{Analysis Method}

\subsection{The Equivalent Circuit Model and Solving Pro- cedure}

As the system is axial symmetrical, the exciting coil and plate are redrawn in $R Z$ plane in Fig. 3. As known, due to the skin effect, the distribution of eddy current is nonuniform. Therefore, to construct an equivalent circuit model that can represent the distribution of eddy current, the plate is divided into many small segments, and in each of which the eddy current density is assumed same as shown in Fig. 3 . The individual segment actually corresponds to a conductive ring with circuit parameters of resistance and inductance. The equivalent circuit model of the system is presented in Fig. 4. Each segment is represented by one circuit loop with parameters of resistance, self inductance and mutual inductances with other circuits. The electromagnetic relationship between the exciting coil and plate is abstracted as the parameter of mutual inductance between exciting coil and each segment. $L_{s}$ is the self inductance of the exciting coil, while $L_{s n}$ is the mutual inductance between the exciting coil and the $n$-th segment.

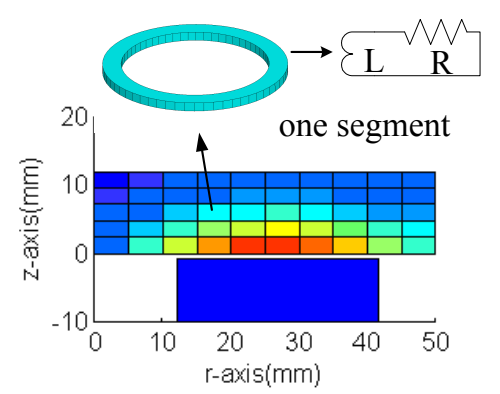

Fig. 3. The exciting coil and plate in $R Z$ plane.

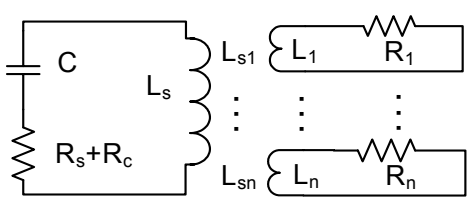

Fig. 4. The equivalent circuit model of the actuator.

The basic calculation procedure of the proposed analytic method is shown as follows:

Step 1 . The plate is initially divided into some segments uniformly and each segment corresponds to one circuit loop with parameters of resistance, self inductance and mutual inductance with other circuits.

Step 2. The self inductance of all segments and the exciting coil as well as the mutual inductance between them is 
calculated. As the mutual inductance is the function of displacement, the mutual inductance curves are constructed by using Bezier interpolation.

Step 3. The circuit equations are combined together with the motion equations to form the electromechanical coupled equations of the system.

Step 4. To use time stepping method, the whole calculation process is divided into multi time steps. At each time step, the circuit parameters are calculated and input into the state equations, and the ordinary differential equations is solved by using Runge-Kutta-Fehlberg method.

Step 5. The dynamic characteristics of the system such as the exciting current, displacement and speed are obtained.

Step 6. According to the calculation result of eddy current density error of each segment, the segments with bigger error will be refined into smaller segments. Then the calculation will return to step 2 . The whole process will not stop until a convergent result is obtained.

\subsection{Governing Equations}

If the plate is divided into $n$ segments, together with the exciting circuit, there are $n+1$ circuits. The equations for the exciting circuit and segments circuits are shown as follows:

$$
\begin{gathered}
Q / C+I_{s} R_{s}+d \lambda_{s} / d t=0 \\
I_{i} R_{i}+d \lambda_{i} / d t=0, i=1,2, \cdots, N \\
\frac{d \lambda_{s}}{d t}=L_{s s} \frac{d I_{s}}{d t}+\sum_{j=1}^{N} L_{s j} \frac{d I_{j}}{d t}+\sum_{j=1}^{N} I_{j} \frac{\partial L_{s j}}{\partial z} \frac{d z}{d t} \\
\frac{d \lambda_{i}}{d t}=L_{s i} \frac{d I_{s}}{d t}+\sum_{j=1}^{N} L_{i j} \frac{d I_{j}}{d t}+I_{s} \frac{\partial L_{s i}}{\partial z} \frac{d z}{d t}, \quad i=1,2, \cdots, N
\end{gathered}
$$

where $R_{s}$ and $\lambda_{s}$ are the resistance and flux linkage of the exciting circuit. $I_{s}$ is the exciting current. $Q$ and $C$ stand for the charge and capacitance. $R_{i}$ and $\lambda_{i}$ are the resistance and flux linkage of the $i$-th circuit. $I_{i}$ stands for the eddy current in the $i$-th circuit. $L_{s i}$ is the mutual inductance between the exciting circuit and $i$-th circuit. $L_{\mathrm{ij}}$ is the mutual inductance between the $i$-th and $j$-th circuits. As there is no magnetic material in the system, the inductance only depends on the geometry dimension, so in (3) the derivative of mutual inductance to time can be written to the derivative to displacement multiplied by the speed of the plate. As the self inductance will not change with the relative position between the exciting coil and the plate, therefore, the derivative of the self inductance to the position is zero. Furthermore, all the segments move together as a whole plate, it is unnecessary to calculate the derivative of mutual inductance between two segments.

If all the inductance and eddy currents are known, the expressions of energy $W$ and electromagnetic force $F_{e m}$ are shown as follows:

$$
\begin{gathered}
W=\frac{1}{2} \sum_{i=s, 1}^{N} \sum_{j=s, 1}^{N} L_{i j} I_{i} I_{j} \\
F_{e m}=-\frac{\partial W}{\partial z}=-\sum_{i=1}^{N} I_{s} I_{i} \frac{\partial L_{s i}}{\partial z}
\end{gathered}
$$

It can be seen that the electromagnetic repulsion force is only decided by the derivative of the mutual inductance between the exciting coil and each segment, as well as the exciting current and the eddy current in each segment.

The motional equations are shown as follows:

$$
\begin{gathered}
\dot{I}_{N+1}=\dot{z}=I_{N+2} \\
\dot{I}_{N+2}=\ddot{z}=\left(F_{e m}-F_{G}\right) / m \\
\dot{I}_{N+3}=\dot{Q}=I_{s}
\end{gathered}
$$

where $I_{N+1}, I_{N+2}, I_{N+3}$ represent the displacement, speed and charge respectively. $F_{G}, m$ are load force and plate mass, respectively.

The dynamic state equations of the system can be obtained by combining (1)-(3) and (6)-(8) as follows:

$$
\begin{gathered}
L_{s s} \dot{I}_{s}+\sum_{j=1}^{N} L_{s j} \dot{I}_{j}=-\frac{Q}{C}-I_{s} R_{s}-I_{N+2} \sum_{j=1}^{N} I_{j} \frac{\partial L_{s j}}{\partial z} \\
L_{s i} \dot{I}_{s}+\sum_{j=1}^{N} L_{i j} \dot{I}_{j}=-I_{i} R_{i}-I_{N+2} I_{s} \frac{\partial L_{s i}}{\partial z}, \quad i=1,2, \cdots, N \\
\dot{I}_{N+1}=I_{N+2} \\
\dot{I}_{N+2}=\left(F_{e m}-F_{G}\right) / M \\
\dot{I}_{N+3}=I_{s}
\end{gathered}
$$

where, $F_{e m}$ can be calculated from (5). The dynamic characteristics can be obtained by solving these ordinary differential equations with initial conditions.

\subsection{Self and Mutual Inductance Calculation}

As mentioned before, the calculation of inductance is one key point to the whole calculation procedure. As the mutual inductance is just the function of structure in the nonmagnetic system, several ways can be adopted to calculate the inductance, for example, using FEM or using Grover's tables; however, to consider the integrality and continuity of the whole program, a method based on Bartky's transformation is chosen to solve the inductance in our problem. By this method the self and mutual inductance of and between any two coaxial circular coils can be calculated precisely.

To improve the efficiency of calculation, the inductance is calculated first before applying the Runge-KuttaFehlberg method. The mutual inductance and mutual inductance derivative curves are interpolated by using Bezier interpolation method.

Mathematically a parametric Bezier curve is defined as follows: 


$$
P(t)=\sum_{i=0}^{n} B_{i} J_{n, i}(t) \quad 0 \leq t \leq 1
$$

where $B_{i}$ is the vertices of Bezier polygon which is obtained from the method based on Bartky's transformation. $J_{n, i}(t)$ is the $i$-th $n$-th order Bernstein basis function expressed as:

$$
J_{n, i}(t)=\left(\begin{array}{c}
n \\
i
\end{array}\right) t^{i}(1-t)^{n-i}
$$

with

$$
\left(\begin{array}{l}
n \\
i
\end{array}\right)=\frac{n !}{i !(n-i) !}
$$

here $n$, the degree of the defining Bernstein basis function and thus of the polynomial curve segment, is one less than the number of vertices of Bezier polygon which is numbered from 0 to $n$.

The derivative of the mutual inductance curve can be interpolated by using the first derivative of Bezier curve, shown as follows:

$$
P^{\prime}(t)=\sum_{i=0}^{n} B_{i} J_{n, i}^{\prime}(t)
$$

where

$$
J_{n, i}^{\prime}(t)=\frac{(i-n t)}{t(1-t)} J_{n, i}(t)
$$

Therefore, by applying the Bezier interpolation, the mutual inductance and derivative of mutual inductance curves are constructed and one example is shown in Fig. 5.

During the calculation, the mutual inductance value and its derivative value will be got by using linear interpolation. Compare to the on-line calculation of inductance, using Bezier interpolation first and then using linear interpolation is proved more efficient.

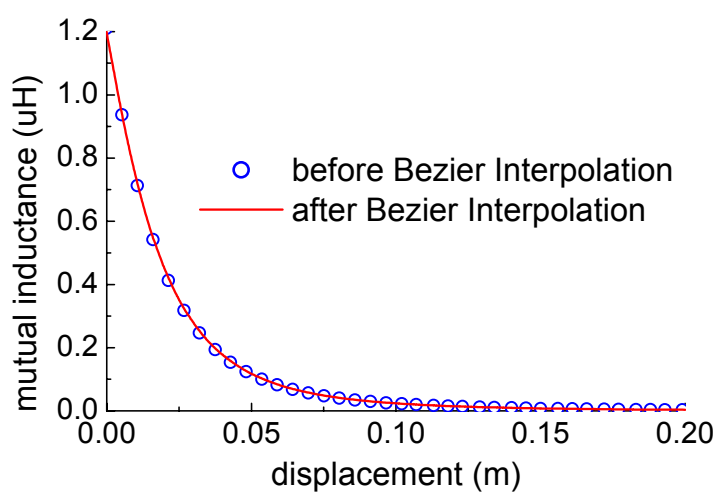

Fig. 5. The mutual inductance varied with displacement.

\subsection{Adaptive Segmentation}

\section{A. Principle of Segmentation}

The accuracy of the solution strongly depends on the segmentation of the conducting plate. Finer segmentation of the conducting plate, in general, gives more accurate performance with more computing time. Hence, in order to achieve precise performance analysis with less computing time, an adaptive segment refinement is developed.

According to the electromagnetic field theory, the tangential component of electric field intensity should be continuous at the interface of two segments. From this, together with Ohm's law, we get, at the interface of segments, the condition of $J_{1 t}=J_{2 t}$. In this paper, local field continuity error for a segment (e), shown in Fig. 6, is defined as follows:

$$
e^{(e)}=\int_{t_{1}}^{t_{2}}\left(\sum_{k=1}^{N_{e}}\left|J(e)-J_{k}\right| \cdot l_{k} / \sum_{k=1}^{N_{e}} l_{k}\right) d t
$$

where $N_{e}$ is the number of the neighboring segments of $e$-th segment, $J^{(e)}$ and $J_{k}$ are the eddy current densities of the $e$ th segment and the $k$-th neighboring segment, respectively, $l_{k}$ is the length of the overlapped interface between $e$-th segment and the $k$-th neighboring segment.

In the first loop, the plate is initially divided into some segments uniformly, after the calculation is finished, the local error for all segments are computed, then the segments with big error will be refined into more segments. Therefore, during the next calculation loop the refined segments will be used instead of the old segments. The calculation will not stop until the result is convergent, which means that the refinement of the plate is reasonable enough to represent the distribution of the eddy current.

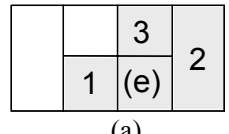

(a)

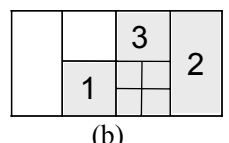

(b)
Fig. 6. Segment refinement. (a) before refinement, (b) after refinement.

\section{B. Segmentation in One Dimension}

To show the segmentation process more clearly, the segmentation is first executed in radial direction. As shown in Fig. 7, the plate is initially divided into four segments along radial direction uniformly. After several calculation loops the plate will be refined more and more, especially in the area where the eddy current density varies sharply. The comparison of the distribution of eddy current density along radial direction of different calculation loops is shown in Fig. 8. It can be easily seen that the initial distribution of eddy current density is quite rough, as the plate is divided into more segments; the distribution of eddy current density turns to a continuous curve. 


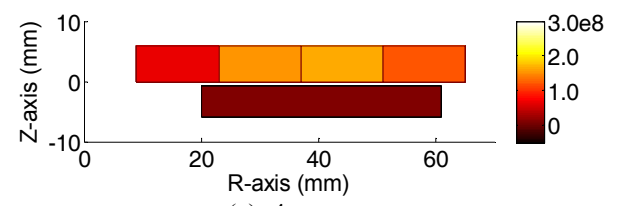

(a) 4 segments

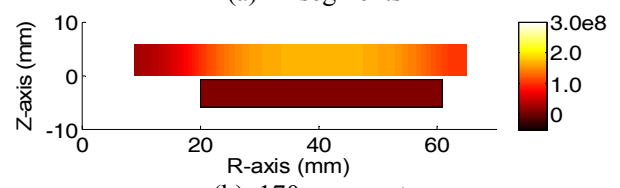

(b) 170 segments

Fig. 7. Division of the plate in radial direction.

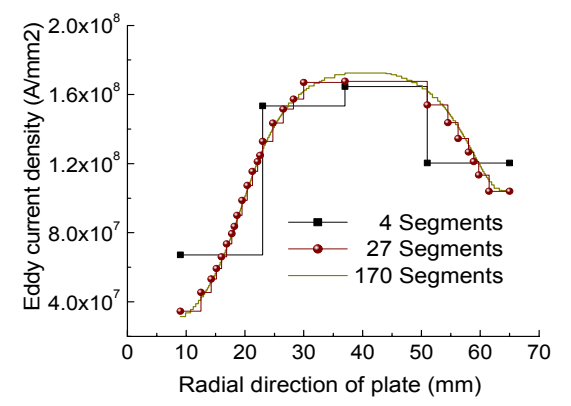

Fig. 8. Comparison of the distribution of eddy current density along radial direction.

\section{Segmentation in Two Dimensions}

The eddy current does not only distribute nonuniformly in radial direction, but also in axial direction. Therefore, the segmentation should be implemented both in radial and axial directions. Fig. 9 shows the segmentation of the plate and distribution of eddy current density in two directions. As the number of segments increases, the distribution of the eddy current density becomes continuous. The skin effect can be shown clearly.

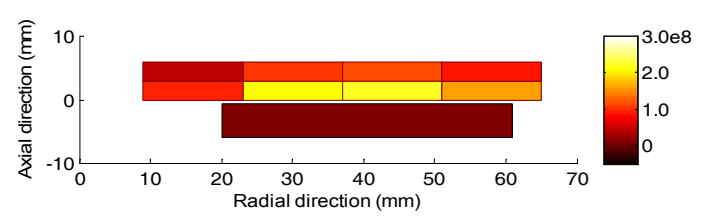

(a) 8 segments

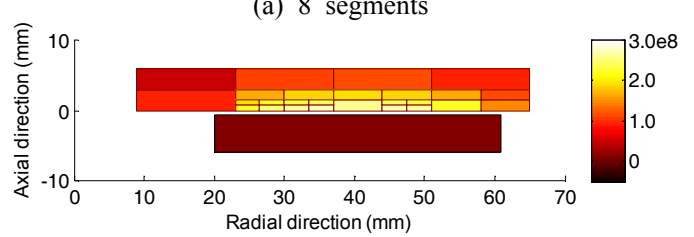

(b) 26 segments

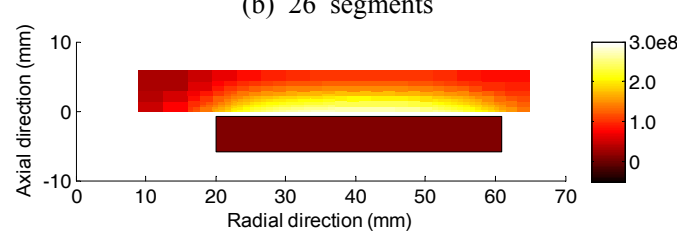

(c) 348 segments

Fig. 9. Distribution of eddy current density and segme ntation of plate in radial and axial directions.

\section{Verification of Result}

As the numerical calculation of the proposed method is based on the segmentation model, the calculation accuracy and efficiency of this solving technique mainly depends on the number of segments and the time step size. The program based on the proposed method is used to analyze the dynamic characteristics of the Thomson coil type actuator which is also solved by using FEM. The winding form of the exciting coil is assumed uniformly, which is in the same situation dealt by FEM. The aluminum and copper are taken as the plate materials respectively. The comparison between the calculation results and experiment results are shown in Fig. 10 and Fig. 11.
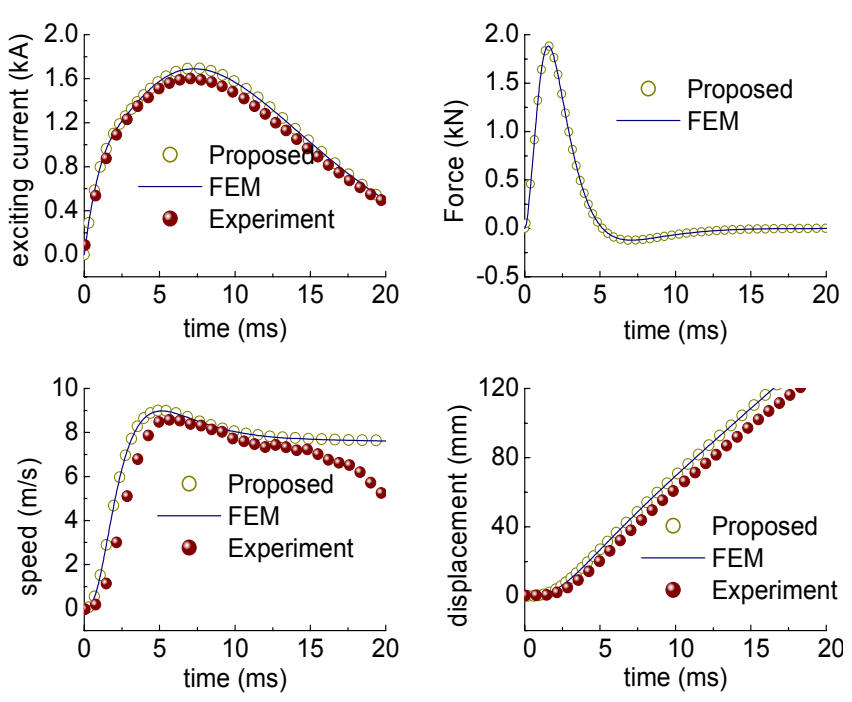

Fig. 10. Comparison of calculation results of Aluminum plate.
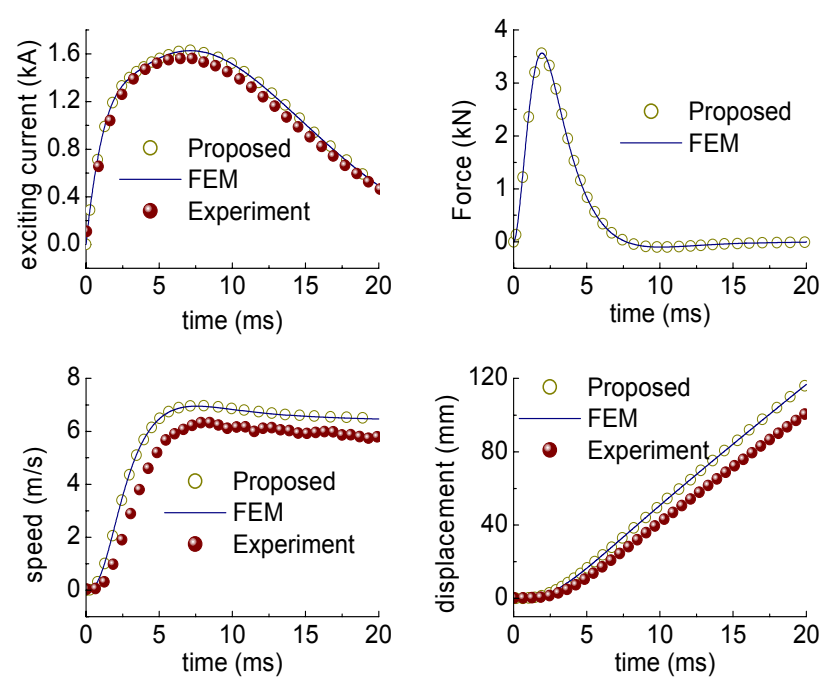

Fig. 11. Comparison of calculation results of Copper plate.

As seen from the results, the calculation results of proposed method match very well with the FEM calculation results for both aluminum and copper plate if the winding 
form of the exciting coil is assumed uniformly. Compare to calculation results, the experiment results show the same changing trend with a tolerant error. This is mainly because the friction that exists in the experiment is not considered in the calculation. Other reasons such as the accuracy of the parameters and the variation of temperature also result in the error.

If the FEM result is taken as the reference, the maximum displacement relative error of the results of proposed method with different number of segments are shown in Table 1. It can be easily seen that with FEM the computing time is about 10 hours, while by adopting the proposed method the computation time is less than 8 minutes even with 94 segments, which is much more efficient while has a good accuracy. Therefore, it is much more convenient and efficient by using the proposed method to analyze and design the high speed Thomson coil type actuator.

Table 1. Comparison of calculation accuracy and efficiency

\begin{tabular}{c|c|c|c}
\hline \multicolumn{2}{c|}{ Calculation method } & $\begin{array}{c}\text { Maximum relative } \\
\text { error (\%) }\end{array}$ & $\begin{array}{c}\text { Computing time } \\
\text { (seconds) }\end{array}$ \\
\hline \multicolumn{2}{c|}{ FEM } & - & 10 hours \\
\hline \multirow{4}{*}{ proposed } & 4 segs & 1.13 & 1.81 \\
\cline { 2 - 4 } & 13 segs & 0.29 & 6.50 \\
\cline { 2 - 4 } & 49 segs & 0.12 & 82.24 \\
\cline { 2 - 4 } & 94 segs & 0.09 & 456.6 \\
\hline
\end{tabular}

\section{Influence of Design Parameters}

If the initial air gap between the exciting coil and plate is fixed to a value, then the parameters that influence the mechanical performance of the Thomson coil type actuator can be labeled as circuit parameters and plate parameters. The circuit parameters include the capacitance, inductance, resistance and the initial voltage of capacitor. Actually, the value of inductance and resistance depend on the parameters of the coil, which correspond to the diameter of the wire, the inner radius of the coil, the number of conductors per layer, and the number of layers, as shown in Fig. 12. The plate parameters specify the plate thickness and the outer radius of the plate. All the parameters are shown in Table 2, as well as the data range.

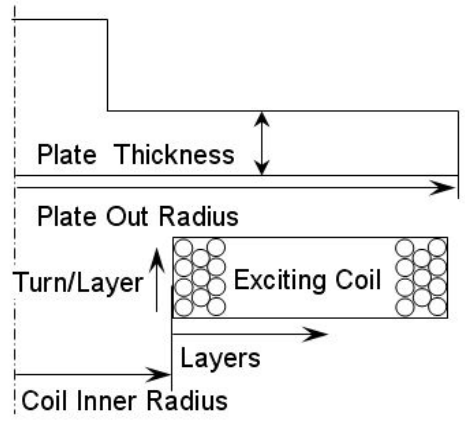

Fig. 12. Design variables of the actuator.
Table 2. Circuit and plate parameters.

\begin{tabular}{c|c|c}
\hline & Parameters & Calculating Points \\
\hline \multirow{4}{*}{$\begin{array}{c}\text { Exciting Cir } \\
\text { cuit }\end{array}$} & Charging Voltage (V) & $100,200,250$ \\
\cline { 2 - 3 } & Capacitor (mF) & $25,50,200,400$ \\
\cline { 2 - 3 } & Number per Layer & $2-4$ \\
\cline { 2 - 3 } & Number of Layers & $10-20$ \\
\cline { 2 - 3 } & Inner Radius (mm) & $15-25$ \\
\cline { 2 - 3 } Plate & Diameter of Coil (mm) & 2.6 \\
\cline { 2 - 3 } & Outer Radius (mm) & $50-80$ \\
\hline
\end{tabular}

To obtain a good performance of the Thomson coil type actuator, which means that get a big displacement in a short time, a set of reasonable parameters values should be selected among the data ranges. From the analysis of properties, it is known that the displacement of the plate is proportional to the exciting current. However, usually the maximum value of the exciting current is limited by electronic switches. Therefore, a reasonable combination of parameters should give a big displacement with a lower exciting current. The calculation related with different combinations of parameters is done by applying the proposed method.

Fig. 13 shows the distribution of calculation results of different parameters combinations, and each point represented by the maximum exciting current and displacement stands for the calculation result of one combination of parameters. Among all the results, the one with bigger displacement and smaller exciting current is preferred. The preferred results are selected and redrawn in Fig. 14. Therefore, to obtain a better performance of the system, the design parameters should be selected among the preferred results.

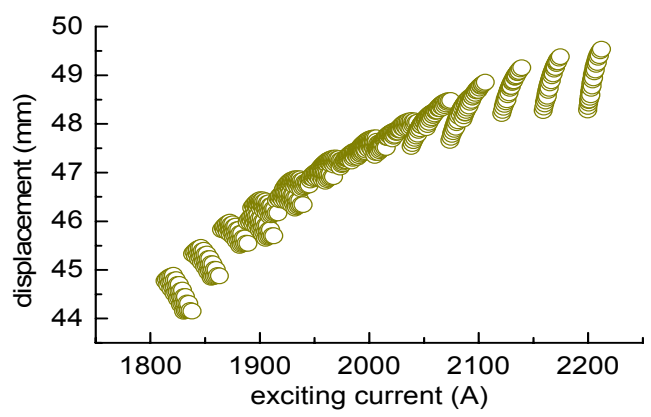

Fig. 13. Calculation results of different parameters combinations.

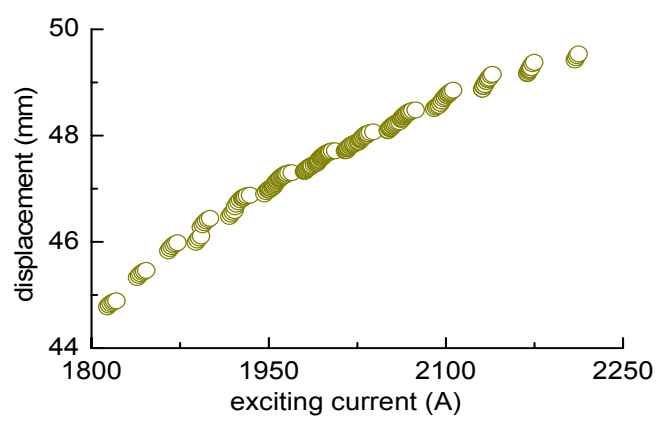

Fig. 14. Preferred calculation results. 
To analyze the influence of parameters, some of the calculation results are redrawn in Fig. 15. From the figure it can be seen that the results distribute in the form of some clusters. Actually, each cluster stands for one combination of circuit parameters, which means that the circuit parameters will decide the position of clusters in the figure. Therefore, a reasonable combination of circuit parameters will result in a good position of the corresponding cluster.

In one cluster, the circuit parameters for all points are same while only the plate parameters are different with each other. One cluster is taken out and redrawn in Fig. 16 and Fig. 17. If the outer radius of the plate is fixed to certain value, then only one parameter, the thickness of the plate, will influence the result, as shown in Fig.16. From the result it can be seen for each curve there is a maximum value when the thickness changes. The points that locate on the left side of the maximum point are reasonable while the one on the right side are not suitable as the displacement decreases when the exciting current increases. In the same way, if the thickness of the plate is fixed to certain value, then only the outer radius of plate will influence the result, the curves are shown in Fig. 17. It can also be seen there exists a maximum value on each curve, the points that locate on the left side of the maximum point are reasonable. Therefore, if the circuit parameters are determined first, there exist best plate parameters which will give a good result.

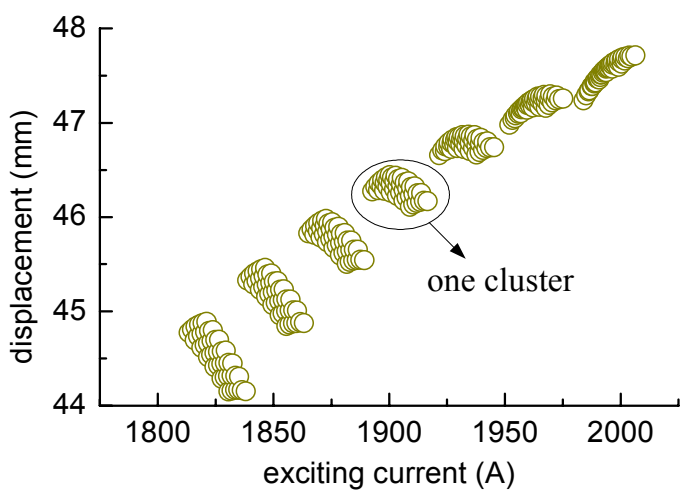

Fig. 15. Calculation results influenced by circuit parameters.

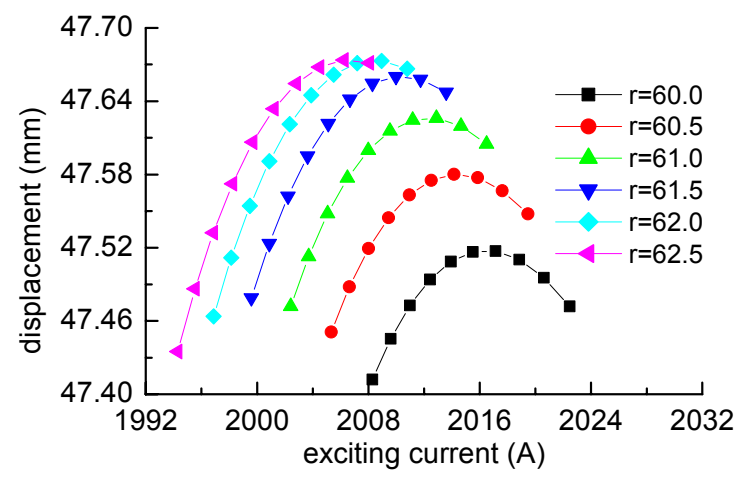

Fig. 16. Calculation results influenced by thickness of plate.

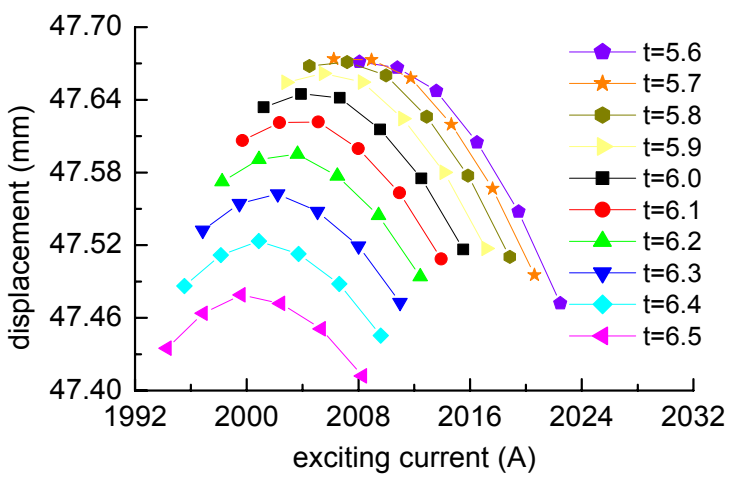

Fig. 17. Calculation results influenced by outer radius of plate.

\section{Conclusion}

The dynamic characteristics of Thomson coil type actuator used in an arc eliminator have been analyzed by using a novel solving technique which is developed based on the equivalent circuit model. Considering the influence of skin effect, the conducting plate is divided into many segments. An adaptive segment refinement algorithm based on the field continuity condition is proposed to get minimum segments number of the conducting plate for precise performance analysis. This solving technique is quite efficient, which is important for designing and analyzing electromagnetic devices. The experiment result is also compared with analysis result, and it shows the same changing trend which proves the correctness of calculation result. The influence of parameters is observed by the calculation with different parameters combinations, through which a set of reasonable design parameters that gives a big displacement with a small exciting current can be found.

\section{References}

[1] Toshie Takeuchi, Kenichi Koyama and Mitsuru Tsukima, "Electromagnetic Analysis Coupled with Motion for High-Speed Circuit Breakers of Eddy Current Repulsion Using the Tableau Approach," Electrical Engineering in Japan, Vol. 152, No. 4, pp. 8-16, 2005.

[2] Seung-Myen Lee, Se-Hee Lee, Hong-Soon Choi and Il-Han Park, "Reduced Modeling of Eddy CurrentDriven Electromechanical System Using Conductor Segmentation and Circuit Parameters Extracted by FEA,” IEEE Trans. on Magn., Vol. 41, No. 5, pp. 1448-1451, May 2005.

[3] Se-Hee Lee, In-Gu Kwak, Hong-Song Choi, SeungMyen Lee, Il-Han Park and Won-Kyu Moon, "Fast solving technique for mechanical dynamic characteristic in electromagnetic motional system by electromechanical state equation including extracted circuit parameter," IEEE Trans. on applied superconductivity, Vol. 14, No. 2, pp. 1926-1929, June 2004. 
[4] Fawzi TH, Burke PE, The accurate computation of self and mutual inductances of circular coils. IEEE Trans Power Apparatus Syst., Vol. 97, No. 2, pp. 464468, March 1978.

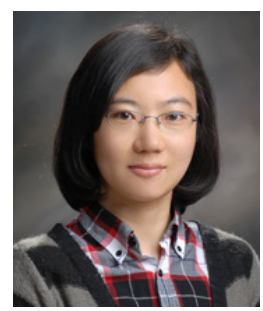

Wei Li received the B.S. and M.S. degrees in Electrical Engineering from Harbin Institute of Technology, Harbin, China, in 2005 and 2007. She is currently working towards $\mathrm{Ph}$. D. degree in department of Electrical Engineering in Chungbuk National University, Korea. Her research interests include optimal design of electromagnetic devices and numerical analysis of electromagnetic fields.

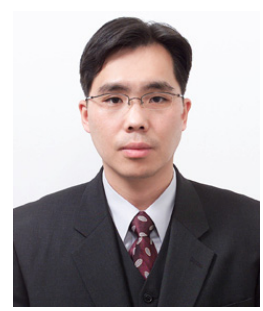

Young Woo Jeong received the B.S. and M.S. degrees in the department of Electrical Engineering from Kon-kuk University, Seoul, Korea, in 1994, 1996 respectively. He was a research engineer at the Technology research center of the Taihan Electric Wire Co., Ltd. from January 1996 to June 2002. He has been a senior research engineer at the Electrotechnology Center of LS Industrial Systems Co., Ltd. since July 2002. His research interests include high-voltage electrical equipment design and analysis and simplified synthetic testing of the electrical switch from MV to UHV.

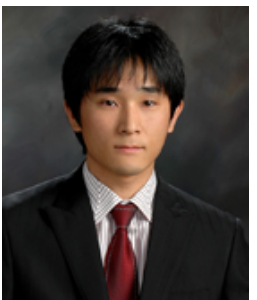

Hee Sung Yoon received the B.S. and M.S. degrees in the department of electrical and electronics engineering, Chungbuk National University in 2005 and 2007. He is currently working towards $\mathrm{Ph}$. $\mathrm{D}$. degree on electric machines. His research interests include numerical analysis of electromagnetic fields and shape optimal design of electromagnetic devices.

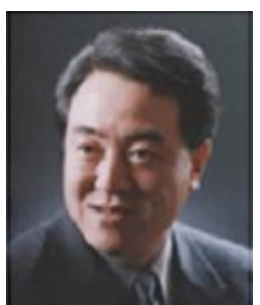

Chang Seop Koh received his B.S., M.S., and Ph. D. degrees in Electrical Engineering from Seoul National University, Seoul, Korea, in 1982, 1986, and 1992, respectively. He was visiting Professor at the Department of Electrical and Computer Engineering, Florida International University, Florida, USA, and at the Department of Electrical and Computer Engineering, Texas A\&M University, Texas, USA, from May 1993 to April 1994, and from February 2003 to January 2004, respectively. He was also a Senior Researcher at the Central Research Institute of Samsung Electro- Mechanics Co., Ltd., from May 1994 to August 1996. He has been a Professor with the School of Electrical and Computer Engineering, Chungbuk National University, Korea, since 1996. His research interests include electric machine design, numerical analysis of electric machines using the finite element and boundary element methods. 